\title{
INFLUENNCIA DO CICLO LUNAR NA FREQUÊNCIA DE ADMISSÕES HOSPITALARES NA MATERNIDADE: ESTUDO RETROSPECTIVO
}

\author{
INFLUENCE OF THE LUNAR CYCLE ON THE \\ FREQUENCY OF MATERNITY HOSPITAL ADMISSIONS: \\ A RETROSPECTIVE STUDY
}

\section{INFLUENCIA DEL CICLO LUNAR EN LA FRECUENCIA DE ADMISIONES HOSPITALARIAS EN LA MATERNIDAD: ESTUDIO RETROSPECTIVO}

\author{
Jessica Torres Grasel ${ }^{1}$ \\ Thamiza Laureany da Rosa dos Reis ${ }^{2}$ \\ Jacqueline Silveira de Quadros ${ }^{3}$
}

Como citar este artigo: Grasel JT, Reis TLR, Quadros JS. Influência do ciclo lunar na frequência de admissões hospitalares na maternidade: estudo retrospectivo. Rev baiana enferm (2018);32:e26537.

\begin{abstract}
Objetivo: analisar se a frequência de admissões hospitalares na maternidade sofre influência do ciclo lunar. Método: estudo analítico, documental, retrospectivo, com abordagem quantitativa, desenvolvido em uma maternidade no Sul do Brasil, no período de julho de 2013 a julho de 2014. A amostra foi composta por 845 prontuários de parturientes. A análise dos dados foi do tipo descritiva e utilizou-se teste exato de Fischer e Qui-Quadrado. Resultados: aproximadamente $84 \%(n=709)$ das mulheres não foram admitidas na maternidade cenário do estudo durante os dias de mudança de Lua e não houve diferença significativa entre o número de admissões em cada fase lunar, determinando $(\mathrm{p}=0,1116)$. Conclusão: evidenciou-se que as mudanças de fase da Lua não foram fatores determinantes para o aumento da demanda de cuidados obstétricos e admissão hospitalar na instituição cenário deste estudo.
\end{abstract}

Descritores: Obstetrícia. Parto. Trabalho de Parto. Lua.

Objective: to analyze whether the frequency of maternity hospitals admissions is influenced by the lunar cycle. Method: an analytical, documentary, and retrospective study with a quantitative approach was carried out in a maternity hospital in the South of Brazil from July 2013 to July 2014. The sample was made up of 845 medical records of pregnant women. A descriptive data analysis was carried out with the use of the Fisher's exact test and chi-square test. Results: approximately 84\% ( $n=709)$ of the pregnant women were not admitted to the maternity hospital setting of the present study during the days of lunar change and no significant difference in the number of admissions in each lunar phase was found, determining $p=0.1116$. Conclusion: it was evidenced that lunar phase changes were not determining factors for the increase in the demand of obstetric care and hospital admission in the institution setting of the present study.

Descriptors: Obstetrics. Parturition. Obstetric Labor. Moon.

Enfermeira obstetra. Hospital Universitário de Santa Maria. Santa Maria, Rio Grande do Sul, Brasil. jessicagrasel@hotmail.com

Enfermeira obstetra. Mestre em Enfermagem. Hospital Escola da Universidade Federal de Pelotas. Pelotas, Rio Grande do Sul, Brasil. thamizal@hotmail.com

Enfermeira obstetra. Doutoranda em Enfermagem pelo Programa de Pós-graduação em Enfermagem da Universidade Federal de Santa Maria. Hospital Universitário de Santa Maria. Santa Maria, Rio Grande do Sul, Brasil. 
Objetivo: analizar si la frecuencia de admisiones hospitalarias en la maternidad recibe influencia del ciclo lunar. Método: estudio analítico, documental, retrospectivo, con abordaje cuantitativo, desarrollado en una maternidad del Sur de Brasil, entre julio de 2013 y julio de 2014. Muestra integrada por 845 historias clinicas de parturientas. Datos estudiados por análisis descriptivo; se utilizó test exacto de Fischer y Chi-cuadrado. Resultados: aproximadamente el 84\% ( $n=709$ ) de las mujeres no fueron admitidas en la maternidad sede del estudio durante los dias de cambio de luna, y no bubo diferencia significativa entre el número de admisiones en cada fase lunar, determinando $(p=0,1116)$. Conclusión: se evidenció que los cambios de fase lunar no constituyeron factores determinantes para incremento de la demanda de cuidados obstétricos y admisión hospitalaria en la institución sede del estudio.

Descriptores: Obstetricia. Parto. Trabajo de Parto. Luna.

\section{Introdução}

A forma como as mulheres percebem os eventos relacionados ao parto e nascimento de seus filhos está diretamente relacionada com saberes e tradições que lhes foram transmitidos ao longo das gerações. Esses mitos, superstições e crenças populares são saberes empíricos, originados de histórias de gestações ou partos relatados por seus familiares e pessoas de sua comunidade, caracterizando étnica, religiosa e culturalmente determinados grupos ${ }^{(1)}$.

Dentre as muitas crenças populares, uma das mais antigas e conhecidas é aquela que relaciona as fases lunares ao processo de parturição. Desde a ancestralidade, diferentes culturas relacionam a Lua à concepção, gestação e especificamente ao parto ${ }^{(2)}$. Para os povos antigos, a luz da Lua é responsável pela germinação e crescimento das plantas e concepção dos animais. Assim, acreditava-se que as mulheres seguiam essa mesma natureza. A conexão entre a mulher e a Lua era tanta que o termo menstruação, em muitas línguas, significa "mudança de Lua".

Mesmo em textos clássicos da obstetrícia, a Lua rege diretamente o ciclo gestacional, uma vez que a duração da gestação é definida em dez meses lunares, ou nove meses solares, ou, ainda, quarenta semanas ${ }^{(4)}$. Essa influência lunar no dia de nascimento criou raízes na cultura popular, envolvendo o misticismo, a astrologia e a ciência. Ainda hoje, essa crença instiga profissionais a relacionarem o aumento da demanda por cuidados obstétricos aos ciclos lunares ${ }^{(2)}$.

Essa relação ocorre comumente por dois motivos. O primeiro, pela crença de que a Lua cheia, comparada às demais fases da Lua, influencia no início do trabalho de parto ${ }^{(2,5)}$. E o segundo, que Lua exerce grande influência sobre os líquidos existentes no planeta, principalmente nos mares. Nessa perspectiva, entende-se que, como o corpo humano é composto por 60\% de água, também sofre influência da Lua. Assim, na gestação, essa influência seria maior, pois o feto está imerso no líquido amniótico ${ }^{(6-7)}$.

Ainda que em pequeno número, estudos com grupos populacionais em diversas regiões do mundo utilizaram diferentes metodologias para correlacionar o processo de parturição às fases lunares, encontrando resultados contraditórios. No que se refere às pesquisas brasileiras, não são encontradas publicações que busquem associações ou comprovações dessa hipótese ${ }^{(8-9)}$.

Estudos dessa natureza podem contribuir com o entendimento do misticismo que envolve a Lua, permeia as maternidades e instiga os profissionais. Além disso, fornece subsídios teóricos para que os profissionais envolvidos no cenário do parto desenvolvam orientações e práticas baseadas em evidências científicas, visando o melhor preparo e qualificação da assistência obstétrica. Neste sentido, têm-se como objetivo analisar se a frequência de admissões hospitalares na maternidade sofre influência do ciclo lunar.

\section{Método}

Estudo analítico, retrospectivo, realizado por meio de levantamento documental, com abordagem quantitativa. O local de pesquisa foi uma 
maternidade localizada no interior do estado do Rio Grande do Sul, Brasil, voltada para o atendimento das situações de risco obstétrico habitual. Por se tratar de uma instituição pública, sua assistência é exclusivamente dirigida às usuárias do Sistema Único de Saúde (SUS).

Os dados foram coletados em prontuários de parturientes de risco obstétrico habitual, assistidas na instituição cenário do estudo, no período de julho de 2013 a junho de 2014, abrangendo 12 ciclos lunares. Entende-se por risco obstétrico habitual: parturientes livres de diagnósticos de patologias maternas, idade gestacional entre 37 e 41 semanas, gravidez única, apresentação fetal cefálica e peso ao nascer adequado para a idade gestacional ${ }^{(10)}$. Tendo em vista que o estudo teve foco na análise da influência do ciclo lunar no desencadeamento do trabalho de parto, foram excluídos os prontuários de mulheres que não entraram em trabalho de parto espontâneo e aquelas que realizaram cesariana eletiva. A amostra total foi composta por 862 prontuários. Destes, houve 17 perdas, pois os prontuários não continham as informações completas relativas à admissão hospitalar. Desse modo, a amostra elegível foi composta por 845 prontuários.

O procedimento de coleta dos dados compreendeu o período entre os meses de agosto e dezembro de 2014, junto ao Serviço de Arquivo Médico do hospital, que forneceu os prontuários. Destes foram extraídas as informações necessárias ao preenchimento do formulário elaborado pelas próprias autoras, no qual constavam: dados de identificação, características sociodemográficas (idade materna, situação conjugal, ocupação e grau de escolaridade), antecedentes obstétricos (número de partos anteriores, idade gestacional, dilatação cervical, dinâmica uterina, estado das membranas amnióticas e tipo de parto) e dados sobre o ciclo lunar (fase lunar e período de mudanças de Lua durante as admissões hospitalares).

Após a coleta de dados, as informações foram digitadas no programa Microsoft Excel 2010 e depois exportadas para o Software Statistical Package for the Social Sciences (SPSS) versão 20.0 para Windows. Realizou-se estatística descritiva para caracterização da amostra, perfil socioeconômico e demográfico das parturientes. Foi utilizado o teste exato de Fischer para avaliar a distribuição das variáveis independentes frente à variável dependente. Também se utilizou o teste Qui-Quadrado (QUI ${ }^{2}$ ) com nível de significância de $5 \%$.

Os dados apresentados fazem parte de uma pesquisa $^{(11)}$ desenvolvida e apresentada no ano de 2015 como requisito parcial para obtenção do título de enfermeira obstetra, aprovada pelo Comitê de Ética em Pesquisa do Centro Universitário Franciscano de Santa Maria, sob o Parecer n. 739.564/2014. Foram respeitados os critérios éticos explicitados nas Diretrizes e Normas Regulamentadoras de Pesquisas envolvendo Seres Humanos aprovadas pela Resolução n. 466/12 do Conselho Nacional de Saúde ${ }^{(12)}$.

\section{Resultados}

Quanto ao perfil sociodemográfico, a população do estudo caracterizou-se por mulheres jovens $(55,4 \%$ [n=468]), com idade entre 15 e 24 anos de idade (18,6\% [n=157]), adolescentes com idade entre 12 e 18 anos. Com relação à cor de pele, 68,8\% (n=581) autodeclarou-se branca, 18,1\% $(n=153)$ parda e $13,1 \%(n=111)$ preta (Tabela 1$)$.

Tabela 1 - Dados sociodemográficos das parturientes de risco obstétrico habitual. Santa Maria, Rio Grande do Sul, Brasil - 2013-2014 (N=845)

\begin{tabular}{|c|c|c|}
\hline Variáveis & $\mathbf{n}$ & $\%$ \\
\hline \multicolumn{3}{|l|}{ Escolaridade } \\
\hline Sem escolaridade & 4 & 0,5 \\
\hline Ensino fundamental ( $1^{\underline{a}}$ a $4^{\underline{a}}$ série) & 52 & 6,1 \\
\hline Ensino Fundamental (5 $5^{\mathrm{a}}$ a $8^{\mathrm{a}}$ série) & 381 & 45,1 \\
\hline Ensino médio e superior incompleto & 382 & 45,2 \\
\hline Ensino superior & 26 & 3,1 \\
\hline
\end{tabular}


Tabela 1 - Dados sociodemográficos das parturientes de risco obstétrico habitual. Santa Maria, Rio Grande do Sul, Brasil - 2013-2014 (N=845) (conclusão)

\begin{tabular}{lcc}
\hline Variáveis & $\mathbf{n}$ & $\mathbf{\%}$ \\
\hline Ocupação & & 39.9 \\
Emprego fixo ou atividades remuneradas & 337 & 46,3 \\
Do lar & 391 & 13,8 \\
Estudantes & 117 & 78,7 \\
Situação conjugal & & 16,4 \\
Solteiro & 665 & 1,7 \\
Casado ou união consensual & 139 & 3,2 \\
Divorciado & 14 & 27 \\
Viúvo & & \\
\hline
\end{tabular}

Fonte: Elaboração própria.

Referente às características clínico-obstétricas no momento da admissão hospitalar, 51,6\% $(n=436)$ das mulheres não se encontravam em trabalho de parto ativo, isto é, não possuíam dinâmica uterina forte e dilatação mínima de quatro centímetros, 41,3\% $(n=349)$ foram admitidas com o colo uterino pérvio entre 4 e 7 centímetros e 5,2\% (n=44) entre 7 centímetros e dilatação completa do colo uterino. No mesmo período, 80,1\% $(n=677)$ das mulheres apresentavam membranas amnióticas íntegras. Dessas 39,4\% (n=267) apresentaram ruptura espontânea de membranas amnióticas durante o trabalho de parto (Tabela 2).

Tabela 2 - Dados clínico-obstétricos das parturientes de risco obstétrico habitual. Santa Maria, Rio Grande do Sul, Brasil - 2013-2014 (N=845)

\begin{tabular}{lcc}
\hline Variáveis & $\mathbf{n}$ & $\mathbf{\%}$ \\
\hline Partos anteriores & & 56.6 \\
Nulípara & 478 & 20,6 \\
Primípara & 174 & 12.4 \\
Secundípara & 105 & 10,4 \\
Multípara (três ou mais partos) & 88 & 7 \\
Idade gestacional no início do trabalho de parto & & 22,8 \\
37 semanas a 37 semanas e 6 dias & 59 & 33,6 \\
38 semanas a 38 semanas e 6 dias & 193 & 36,6 \\
39 semanas a 39 semanas e 6 dias & 284 & 82,4 \\
40 semanas a 40 semanas e 6 dias & 309 & 17,7 \\
Tipo de parto & & 695 \\
Parto normal & 150 & \\
Cesariana de urgência & & \\
\hline
\end{tabular}

Fonte: Elaboração própria.

As indicações mais frequentes para a realização de cesariana relatadas nos prontuários da população estudada foram condição fetal não tranquilizadora, desproporção céfalo-pélvica, parada de progressão do trabalho de parto e posição pélvica. A respeito das associações entre os ciclos lunares e a admissão hospitalar, os dados apontaram que aproximadamente 84\% ( $n=709)$ das mulheres não foram admitidas na maternidade cenário do estudo durante os dias de mudança de Lua. Sobre os ciclos lunares, não houve diferença significativa entre o número de admissões em cada fase lunar ( $\mathrm{p}=0,1116)$ (Tabela 3). 
Tabela 3 - Distribuição das admissões hospitalares segundo fase do ciclo lunar. Santa Maria, Rio Grande do Sul, Brasil - 2013-2014 (N=845)

\begin{tabular}{lcc}
\hline Admissão hospitalar & n & $\mathbf{\%}$ \\
\hline Mudança de fase da Lua & & \\
$\quad$ Dias de mudança de fase da Lua & 138 & 16,3 \\
$\quad$ Dias comuns & 707 & 83,7 \\
Fases da Lua & & \\
$\quad$ Nova & 221 & 26,2 \\
Quarto-Crescente & 226 & 26,7 \\
$\quad$ Cheia & 186 & 22 \\
Quarto-Minguante & 212 & 25,1 \\
\hline
\end{tabular}

Fonte: Elaboração própria.

Da totalidade de mulheres deste estudo, 51,4\% (n=435) apresentaram ruptura espontânea de membranas amnióticas, seja anterior à admissão hospitalar 19,8\% (n=168), seja durante a internação hospitalar 31,5\% (n=267). Quando considerada a relação entre a ruptura espontânea e os dias de mudança da fase lunar, os dados estatísticos demonstraram que 36,8\% $(n=160)$ das rupturas espontâneas ocorreram durante a mudança da fase da Lua e 63,2\% (n=275), em dias comuns. Em relação às diferentes fases lunares, não houve diferença entre as proporções de bolsa íntegra e rota $(\mathrm{p}=0,8006)$.

\section{Discussão}

O perfil social e obstétrico das mulheres em estudo caracterizou-se por jovens, nulíparas, na sua grande maioria: solteiras, com baixo nível escolar e exercendo atividades em ambiente domiciliar. Acredita-se que o alto quantitativo de mulheres que relataram não exercer ocupação remunerada esteja relacionado, além da idade, à baixa escolaridade verificada. Percebe-se que, para as mulheres jovens, o casamento e a maternidade ainda são os eventos mais importantes na sua inserção social, muito embora a sua participação na escola e no mercado de trabalho tenha crescido ${ }^{(13)}$.

Constatou-se grande número de admissão de mulheres na fase latente de trabalho de parto. Da população estudada, aproximadamente a metade das mulheres foi internada em momento oportuno, isto é, início da fase ativa do trabalho de parto. Reconhecidamente, a admissão hospitalar precoce aumenta o tempo de internação da parturiente, submetendo-a ao ambiente hospitalar sem necessidade e potencializando o número de intervenções obstétricas, como a rotura precoce das membranas amnióticas e a infusão endovenosa de ocitocina, alterando, assim, o curso fisiológico do parto ${ }^{(14-15)}$.

Quanto ao tipo de parto, observou-se a predominância de partos normais, fato justificado pela clientela da instituição, que se configura como referência regional no atendimento de partos de risco obstétrico habitual. A taxa de cesarianas foi de aproximadamente 18\% ( $n=152)$, não se distanciando dos 10\% a 15\% recomendados pela Organização Mundial de Saúde para todas as regiões do mundo, visando melhores resultados maternos e neonatais, considerando os recursos de saúde disponíveis e as preferências maternas ${ }^{(16-17)}$.

Em relação à associação entre o número de admissões hospitalares e a mudança de fases da Lua, apenas 16\% $(n=135)$ das mulheres foram admitidas durante os dias de transição lunar, evidenciando que esse fator não foi determinante para o aumento de admissões hospitalares na instituição cenário deste estudo. Este fato é reforçado pelos dados, que demonstraram não existir relação entre a mudança de fases da Lua e a proporção de rupturas espontâneas de membranas amnióticas. Esse resultado desmistifica, assim, a crença de que dias de mudança de fase da Lua resultam em maior demanda de cuidados obstétricos. 
Os dados obtidos no presente estudo são similares aos encontrados em pesquisas internacionais. $\mathrm{Na}$ Índia, um estudo retrospectivo e observacional realizado em 9.890 partos vaginais espontâneos não encontrou qualquer associação de efeito lunar no número de nascimentos ${ }^{(2)}$. Da mesma forma, estudos realizados no Brasil, no Peru e na Espanha observaram, respectivamente, 1.122, 1.836 e 5.421 nascimentos e concluíram que não existem provas suficientes para afirmar a relação entre a fase lunar e o início do trabalho de parto $^{(5,18-19)}$.

As fases da Lua resultam do fato de ela não ser um corpo luminoso, e sim um corpo iluminado pela luz do Sol. Suas facetas variam gradualmente, à medida que ela orbita ao redor da Terra ao longo do mês, completando seu ciclo em aproximadamente 29,5 dias. Assim, a face iluminada da Lua é aquela que está voltada para o Sol como também para a Terra. Tradicionalmente, há quatro fases do ciclo: Lua nova, quarto-crescente, Lua cheia e quarto-minguante ${ }^{(7,20)}$.

A observação das diferentes fases lunares, principal objeto de investigação deste estudo, não revelou, nas análises estatísticas, qualquer correlação significativa no número de admissões hospitalares. Nos quatro ciclos lunares, a porcentagem de admissões hospitalares foi similar, entre 22\% e 26\%. Esses números aproximam-se dos resultados encontrados em dois estudos espanhóis ${ }^{(3,19)}$ e vão ao encontro de achados que afirmam não existir uma distribuição específica de partos de acordo com o ciclo lunar, não havendo diferença significativa entre o número de nascimentos nas distintas fases da Lua ${ }^{(2,18-19,21)}$.

Quando comparadas, percebeu-se ainda que não houve diferença estatística significativa no número de admissões hospitalares entre as fases de Lua cheia e as demais fases lunares, mesmo considerando os dias antes e após a mudança da fase lunar. Tal resultado desmistifica a crença de que a Lua cheia é a fase mais importante na indução do início de trabalho de parto e consequentemente maior número de nascimento. Na tentativa de validar essa crença, estudos ${ }^{(2-3,19,21)}$ apresentaram resultados semelhantes, evidenciando que não existe nenhuma diferença estatística entre a quantidade de partos na Lua cheia, seja por via vaginal ou por cesárea.

A superstição relacionada à Lua cheia está baseada na astrologia, uma vez que, durante essa fase lunar, há o alinhamento do Sol com o planeta Terra, e a Lua interfere fortemente no volume, fluxo e refluxo dos líquidos terrestres e nos fluidos presentes no corpo humano. Assim, mesmo que o feto esteja imerso em pequeno volume de líquido amniótico, ele receberia empuxo lunar para ruptura da bolsa amniótica e início do trabalho de parto. Outro fato seria relativo à luminosidade das noites de Lua cheia, o que proporcionaria maior segurança no parto. Curiosamente, ainda hoje uma das recomendações do movimento de humanização do parto é a baixa luminosidade nas salas de parto, a fim de garantir maior conforto e relaxamento da parturiente durante o trabalho de parto. Defende-se ainda os benefícios ao feto, uma vez que ele viveu em um ambiente escuro durante toda a gestação ${ }^{(6,17)}$.

Entretanto, evidenciou-se que não houve diferença entre as proporções de bolsa íntegra e rota nas diferentes fases lunares. Isto é, o fato de ser Lua cheia ou qualquer outra Lua não interferiu no número de bolsas rotas. As pesquisas desenvolvidas nessa perspectiva têm o propósito de investigar a influência das diferentes fases da Lua no início do trabalho de parto. No entanto, não encontraram resultados estatisticamente significativos, refutando essa hipótese ${ }^{(3,7)}$.

Como limitações do estudo, considera-se o fato de ter sido realizado em um hospital escola, marcado pelo uso de intervenções obstétricas, como, por exemplo, a amniotomia e o uso de ocitocina exógena, bastante presentes neste estudo. Acredita-se ainda que exista uma série de fatores relacionados aos sinais de trabalho de parto, admissão hospitalar e ruptura de membranas amnióticas, podendo influenciar nos resultados encontrados. 


\section{Conclusão}

Os achados deste estudo permitiram concluir-se que a mudança de fases da Lua não foi fator determinante para o aumento da demanda de cuidados obstétricos e admissão hospitalar na instituição cenário deste estudo. Demonstrou-se também que não houve diferença significativa no número de admissão relacionada às distintas fases da Lua.

Apesar de não haver nenhuma comprovação científica que embase o misticismo envolvendo a Lua, não é possível desconsiderar seu poder sobre o imaginário das pessoas. Observa-se que esse mito até hoje permeia as maternidades, perpetuando-se na cultura e nas crenças em saúde que envolvem o processo de parir, determinando, por vezes, comportamentos de usuários e de profissionais e interferindo nos cuidados e nas boas práticas. Embora este estudo tenha sido realizado, acredita-se que a superstição que relaciona Lua e aumento do número de partos continuará a existir, o que reforça a necessidade da realização de novos estudos, a fim de desconstruir superstições e concorrer para conclusões mais consistentes, que possam contribuir para a qualificação da assistência obstétrica.

\section{Colaborações:}

1. concepção, projeto, análise e interpretação dos dados: Jessica Torres Grasel e Thamiza Laureany da Rosa dos Reis;

2. redação do artigo e revisão crítica relevante do conteúdo intelectual: Jéssica Torres Grasel, Thamiza Laureany da Rosa dos Reis e Jacqueline Silveira de Quadros;

3. aprovação final da versão a ser publicada: Jéssica Torres Grasel, Thamiza Laureany da Rosa dos Reis e Jacqueline Silveira de Quadros.

\section{Referências}

1. Campos AS, Almeida, ACCH, Santos RP. Crenças, mitos e tabus de gestantes acerca do parto normal. Rev Enferm UFSM [Internet]. 2014 [cited 2015 Oct 16];4(2):332-41. Available from: http://cascavel. ufsm.br/revistas/ojs-2.2.2/index.php/reufsm/ article/view/10245

2. Brarati S, Sarkar M, Haldar PS, Jana S, Mandal S. The effect of the lunar cycle on frequency of births: a retrospective observational study in Indian population. Indian J Public Health [Internet]. 2012 [cited 2015 Dec 16];56(2):152-4. Available from: https://www.ajog.org/article/ S0002-9378(05)00005-0/fulltext

3. Sánchez MVG, Mata AIG, Peñarrocha MGS, Garcia AF, Molina AM. Influencia lunar y barométrica sobre los partos y la rotura espontánea de membranas ovulares. Rev Enferm Albacete [Internet]. 2010 [cited 2015 Dec 16];14:511. Available from: https://www.uclm.es/ab/ enfermeria/revista/numero\%2014/influencia_ lunar_y_barom\%E9trica_s.htm

4. Rezende J. Obstetrícia. 13a ed. Rio de Janeiro: Guanabara Koogan; 2014.

5. Suclla VJÁ, Vásquez DR, Pérez CS, Vela LA. The effect of the lunar cycle on frequency of births: our experience in Peru. Indian J Public Health [Internet]. 2013 [cited 2016 Feb 11];57(3):181-2. Available from: http://www.ijph.in/article.asp?issn=0019557 X; year $=2013 ;$ volume $=57 ;$ issue $=3 ;$ spage $=181 ;$ ep age $=182 ;$ aulast $=$ Suclla

6. Vale NB, Vale LFB, Cruz JR. O tempo e a anestesia obstétrica: da cosmologia caótica à cronobiologia. Rev Bras Anestesiol [Internet]. 2009 [cited 2015 Dec 16];59(5). Available from: http://www.scielo.br/scielo.php?script $=$ sci arttext\&pid=S003470942009000500013\&lng=en

7. Ortiz MY, Suares RIC, Salazar EGR, Ontiveros MCC. Influencia barométrica lunar en la ruptura prematura de membranas en parturientas del Hospital Materno-Infantil Germán Urquídi. Gac Med Bol. 2013;36(1):31-4.

8. Bueno A, Iessi IL, Damasceno DC. Influência do ciclo lunar no parto: mito ou constatação científica? Rev bras enferm [Internet]. 2010 [cited 2015 Nov 15];63(3):477-9. Available from: http://www. scielo.br/scielo.php?script $=$ sci_arttext $\&$ pid $=$ S0034$71672010000300021 \& \operatorname{lng}=\mathrm{en}$

9. Paz ED, Vallina AC, Holgado AA, Lazuén BB. La influencia de las fases lunares en el inicio de parto espontáneo. Nuber Científ [Internet]. 2014 [cited 2015 Dec 16];2(13):58-62. Available from: http:// enfermeriacantabria.com/enfermeriacantabria/ web/articulos/13/108 
10. Leal MC, Pereira APE, Domingues RMSM, Theme Filha MM, Dias MAB, Nakamura-Pereira M, et al. Intervenções obstétricas durante o trabalho de parto e parto em mulheres brasileiras de risco habitual. Cad Saúde Pública. 2014;30(supl 1):17-32.

11. Grasel JT. Influência do ciclo lunar no trabalho de parto: mito ou verdade? [monografia]. Santa Maria (RS): Centro Universitário Franciscano, Residência em Enfermagem Obstétrica; 2015.

12. Brasil. Conselho Nacional da Saúde. Resolução n. 466, de 12 de dezembro de 2012. Aprova as diretrizes e normas regulamentadoras de pesquisas envolvendo seres humanos. Brasília; 2012.

13. Camarano AA, Kanso S. O que estão fazendo os jovens que não estudam, não trabalham e não procuram trabalho? IPEA [Internet]. 2012 [cited 2015 Nov 15];53:37-44. Available from: http://ipea.gov.br/agencia/images/stories/PDFs/ mercadodetrabalho/bmt53_nt03_jovens

14. Backes MTS, Ribeiro LN, Amorim TS, Vieira BC, Souza J, Dias H, et al. Desafios da gestão do cuidado de enfermagem para a qualidade da atenção obstétrica e neonatal em maternidades públicas brasileiras. Invest Qualit Saúde [Internet]. 2017 [cited 2018 Mar 18];2:411-20. Available from: https://proceedings.ciaiq.org/index.php/ ciaiq2017/article/view/1232

15. Leal MC. Parto e nascimento no Brasil: um cenário em processo de mudança. Cad Saúde Pública [Internet]. 2018 [cited 2018 Mar 18];34(5):e00063818. Available from: http://www. scielo.br/scielo.php?script=sci_arttext\&pid=\$0102311X2018000500101\&lng=en
16. Leão MRC, Riesco MLG, Schneck CA, Angelo M. Reflexões sobre o excesso de cesarianas no Brasil e a autonomia das mulheres. Ciênc saúde coletiva [Internet]. 2013 [cited 2015 Nov 15];18(8):2395-400. Available from: http://www. scielo.br/scielo.php?script=sci_arttext\&pid=S1413$81232013000800024 \& \operatorname{lng}=$ en

17. Marcolin AC. Até quando o Brasil será conhecido como o país da cesárea? Rev Bras Ginecol Obstet [Internet]. 2014 [cited 2015 Dec 16];36(7):283-9. Available from: http://www.scielo.br/scielo.php?script=sci arttext\&pid=S0100-72032014000700283

18. Torres AP, Souto AS. Influência das fases da lua sobre os nascimentos. Mito ou verdade? Bol Curso Med UFSC [Internet]. 2018 [cited 2018 Mar 18];1(4). Available from: http://ojs.sites.ufsc.br/index.php/ medicina/article/view/2496

19. Soto HV, Postigo LP, Rubio CJ, Ruda MA, Bru EC, Castellón CA. Influencia de las fases lunares en el inicio del parto, en el Hospital Universitario Virgen de la Arrixaca. Enfermería Global [Internet]. 2007 [cited 2015 Dec 16];11. Available from: http:// revistas.um.es/eglobal/article/view/343

20. Lin JYL, Mora RS. El ciclo lunar y su relación con la distribución de partos en el Hospital de la Mujeres Dr. Adolfo Carit Eva en el año 2004: mito o realidad. Rev med Costa Rica Centroam. 2017;64(581):203-6.

21. Kepler SO, Saraiva MFO. Astronomia e Astrofísica. 3a edição. Porto Alegre: Livraria da Física; 2014.

Recebido: 18 de maio de 2018

Aprovado: 27 de agosto de 2018

Publicado: 26 de novembro de 2018

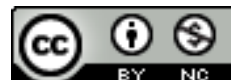

A Revista Baiana de Enfermagem utiliza a Licença Creative Commons - Atribuição-NãoComercial 4.0 Internacional.

https://creativecommons.org/licenses/by-nc/4.0/

Este artigo é de acesso aberto distribuído sob os termos da Licença Creative Commons (CC BY-NC).

Esta licença permite que outros remixem, adaptem e criem a partir do seu trabalho para fins não comerciais. Embora os novos trabalhos tenham de lhe atribuir o devido crédito e não possam ser usados para fins comerciais, os usuários não têm de licenciar esses trabalhos derivados sob os mesmos termos. 